KLEIST-JAHRBUCH 1993 


\section{KLEIST-JAHRBUCH 1993}

Herausgegeben von

Hans Joachim Kreutzer

VERLAG J. B. METZLER STUTTGART · WEIMAR 
Anschrift des Herausgebers:

Universität Regensburg, Institut für Germanistik

D-93040 Regensburg

Redaktion: Sabine Doering

Die Deutsche Bibliothek - CIP-Einheitsaufnabme

Kleist-Jahrbuch ... /-Stuttgart: Metzler.

Erscheint jährlich. - Früher im Verl. E. Schmidt, Berlin. -

Aufnahme nach 1990 (1991)

ISSN 0722-8899

1990 (1991) -

Verl.-Wechsel

ISBN 978-3-476-00953-1

ISBN 978-3-476-03502-8 (eBook)

DOI $10.1007 / 978-3-476-03502-8$

Dieses Werk einschließlich aller seiner Teile ist urheberrechtlich geschützt.

Jede Verwertung außerhalb der engen Grenzen des Urheberrechtsgesetzes ist ohne Zustimmung des Verlages unzulässig und strafbar. Das gilt insbesondere für Vervielfältigungen, Übersetzungen, Mikroverfilmungen und die Einspeicherung und Verarbeitung in elektronischen Systemen.

(C) 1993 Springer-Verlag GmbH Deutschland Ursprünglich erschienen bei Verlag J. B. Metzler, Stuttgart - Weimar und Carl Ernst Poeschel Verlag GmbH in Stuttgart 1993 


\section{INHALT}

Vorwort

VII

\section{Kleist-Preis 1992}

Hans JoAChim KReUtZer: Kein anderer K.! Rede zur Verleihung des Kleist-

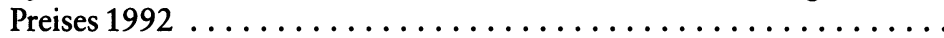

Marcel Reich-Ranicki: Keine Frucht ohne Schale. Rede bei der Verleihung des Kleist-Preises 1992 an Monika Maron . . . . . . . . . . . . .

Monika MaRon: Nach Maßgabe meiner Begreifungskraft .......... 16

Jahrestagung 1992: Zeitenwende $1806-$ Kleists Dichtung im Ausgang des Alten Reiches

WALTER ANNuß (Regensburg): Grußwort der Stadt Regensburg . . . . . . . 23

Helmut Altner (Regensburg): Grußwort der Universität . . . . . . . . 25

HANS JoACHIM KREUTZER (Regensburg): Eröffnung der Jahrestagung 1992 . . 27

Volker Press (Tübingen): Das Ende des Alten Reiches und die deutsche

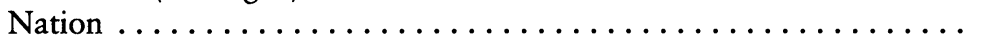

Gerhard Schulz (Melbourne): Von der Verfassung der Deutschen. Kleist und der literarische Patriotismus nach $1806 \ldots \ldots \ldots \ldots \ldots$

HANS-JüRgen BeCKer (Regensburg): Adoption - Verlöbnis - Ehe. Die zivilrechtliche Einbindung des Individuums bei Kleist . . . . . . . . .

Jochen Schmidt (Freiburg i.Br.): Stoisches Ethos in Brandenburg-Preußen und Kleists $>$ Prinz Friedrich von Homburg $\ldots \ldots \ldots \ldots \ldots \ldots \ldots$

RUth KlÜGER (Irvine): Die andere Hündin-Käthchen . . . . . . . . . . 103

Friedmar Apel (Paderborn): »... nur ich allein ging leer aus «. Kleist und die religiös-patriotische Kunstkonzeption ............... 


\section{Abhandlungen}

Roger PAulin (Cambridge): 1806/07 - ein Krisenjahr der Frühromantik? . . . 137

GüNTER Hess (Würzburg): »Durch Adams Fall ist ganz verderbt ...«. Richter Adams Morgenlied . . . . . . . . . . . . . . . . . . . . . . 152

VolKer NöLle (Zürich): Verspielte Identität. Eine expositorische »Theaterprobe « in Kleists Lustspiel `Amphitryon ‘ . . . . . . . . . . . . . . . 160

REGINA SCHÄFER (Szeged): Der gefälschte Brief. Eine unkonventionelle Hypothese zu Kleists ${ }_{\text {Hermannsschlacht }} \ldots \ldots \ldots \ldots \ldots \ldots \ldots \ldots \ldots 1$

Gero von Wilpert (Sydney): Die verabredete Dämmerung . . . . . . . . . 190

\section{Rezensionen}

Gerhard Schulz (Melbourne) über: Peter Schünemann: Die Nacht . . . . . . 197

ANTHony Stephens (Adelaide) über: Timothy J. Mehigan: Text as contract . . 199

Monika Ritzer (Erlangen) über: Hartmut Reinhardt: Apologie der Tragödie, und über: Volker Nölle: Hebbels dramatische Phantasie . . . . . . . . 202

JöRG SCHÖNERT (Hamburg) über: Klaus Lüderssen: Produktive Spiegelungen 210

Hans Joachim KReUtZer (Regensburg): Rechenschaftsbericht 1992 . . . . 216

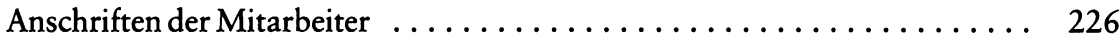




\section{VORWORT}

Der hier vorgelegte Band des Kleist-Jahrbuchs stellt in seinem Hauptteil die Resultate der öffentlichen Jahrestagung 1992 der Heinrich-von-Kleist-Gesellschaft im Druck für die Allgemeinheit zur Diskussion. Diesen Resultaten ist in einem weiteren Sinne auch die Vergabe des Kleist-Preises zuzurechnen. Der nunmehr noch hinzutretende Vortrag von Roger Paulin ist im Planungszusammenhang der gleichen Tagung entstanden. Der Leiter des Department of German der Universität Cambridge sah sich, unvermutet aufgetretener dienstlicher Verpflichtungen wegen, gehindert, an der Tagung teilzunehmen. Der Vortrag wurde ein Jahr später in Regensburg nachgeholt und im Rahmen einer Vortragsreise auch an einigen weiteren Universitäten gehalten. Die in diesem Band folgenden Abhandlungen haben ein gemeinschaftliches Zentrum, indem sie Fragen aus den an der Oberfläche manchmal unlogisch erscheinenden und in ihren Tiefenschichten oft verunsichernden Kleistschen Texten heraus entwickeln. Der Band 1993 des Kleist-Jahrbuchs steht in dem üblichen Rhythmus von Veranstaltung und Publikation. Er erscheint zu einem Zeitpunkt, zu der der - 1992 neu gewählte - Präsident zusammen mit dem Vorstand der Heinrich-von-Kleist-Gesellschaft die Planung der nächsten Jahrestagung (1994) im wesentlichen abgeschlossen hat; sie soll in Frankfurt/Oder stattfinden.

Mit Bedauern ist zu vermelden, daß Professor Shiro Nakamura von der Tohoku Universität Sendai sich aus Gesundheitsgründen genötigt gesehen hat, die von ihm herausgegebenen Kleistblätter NiNGYOSHIBAI (Marionettentheater) nunmehr einzustellen. Nakamura hat sehr große Verdienste um die Nachwirkung Kleists. Seine Zeitschrift, in Japan allgemein beachtet, bildete die wichtigste Vermittlungsbrücke sowohl für die Kleist-Forschung innerhalb Japans wie auch zwischen den japanischen Germanisten und deutschen Kleist-Forschern. Fast jedes der sieben seit 1985 erschienenen Hefte enthielt auch einen Beitrag in deutscher Sprache. $\mathrm{Zu}$ dem im Sommer 1993 erschienenen letzten (1991/92) haben Mishio Suzuki, Shuichi Ito, Ozamu Watanabe, Yoshiro Matsuzawa und Shiro Nakamura Aufsätze beigesteuert. Ein Gesamtinhaltsverzeichnis 1985-1992 bildet den Beschluß (S. 88-90). Shigeru Fukami hat die auf Privatinitiative beruhende Leistung der Kleist-Gesellschaft Sendai im Kleist-Jahrbuch (1990, S.167-169) gewürdigt. Gemeinsam mit meinem Freunde Nakamura hege ich die Hoffnung, daß jüngere japanische Germanisten eine neue Form der Kleist-Pflege in Japan finden werden, sowohl institutionell wie in Publikationen.

Drei Jahre sind vergangen, seitdem der Sowjetstern auch über unserem Lande erloschen ist. Dem politischen Wandel folgt der wissenschaftliche eher zögernd: In den Publikationsorganen der Geisteswissenschaften finden sich bislang nur wenige 
Anzeichen der eingetretenen Veränderungen, das Kleist-Jahrbuch bildet dabei keine Ausnahme. Neue oder auch nur zusätzliche Impulse aus den neuen Territorien des Bundesgebiets sind noch nicht zu erkennen. Man würde sie bei dem Thema Kleist vielleicht sogar eher als auf anderen Gebieten erwarten, weil die Kleistforschung innerhalb des Universitätsfaches Deutsche Philologie traditionell mit den östlichen Provinzen Deutschlands eng verbunden war, Berlin ist geschichtlich, wenn auch nur bis zum Ende des Dritten Reiches, ihr eigentliches Zentrum gewesen. Doch geschichtliche Kontinuität existiert offensichtlich nicht, und gewachsene Tradition wird sogar aufgegeben. Die Berliner Staatsbibliothek hat zwei Kleist-Autographe von völlig unvergleichlichem Wert nicht erworben, trocken gesagt. Damit hat sie eine weit über ein Jahrhundert hingewahrte Kontinuität ihrer Sammlungstätigkeit in Sachen Kleist beendet. Für finanzielle Entscheidungen dieser Größenordnung ist die Stiftung PreuBischer Kulturbesitz direkt verantwortlich. Die im Kleist-Jahrbuch erfolgte Würdigung der außerordentlichen Verdienste der Bibliothek (vgl. Jahrgang 1990, S. 193) ist dahingehend zu präzisieren, daß sie sich auf die Vergangenheit bezieht. Vor dem Hintergrund dieses spektakulären Verlustes an Tradition stellt sich auch die Frage, wie man es in Berlin künftig mit der Kleistforschung überhaupt halten wird. Mit nunmehr drei Universitäten existiert dort ein quantitativ sehr großes Wissenschaftlerpotential. Allerdings ist das West-Berliner Subventionszeitalter zu Ende. In der allgemeinen finanziellen Bedrängnis haben sich die Berliner Universitäten zunächst einmal aneinander zu messen. Eine solche Situation könnte durchaus auch produktiv machen.

In Berlin ist im ablaufenden Jahr unter dem Stichwort Kleist ein Thema aufgebracht, vielleicht geradezu herbeigeredet worden: die im Besitz der Amerika-Gedenkbibliothek in Berlin befindlichen Notizen und sonstigen Nachlaßmaterialien des letzten Präsidenten der 1945 untergegangenen Kleist-Gesellschaft, Georg MindePouet. Dürfte man einigen Journalisten glauben, so hätte man es hier mit einer Art von Nibelungenhort der Kleistforschung zu tun, und die Entdeckung dieses Rheingolds erfolge erst jetzt. Die Beschaffenheit, der Wert und der mögliche Umgang mit diesem Nachlaß sollten in der gebotenen Sachlichkeit beschrieben werden. Die fraglichen Papiere rechnen schon seit Jahrzehnten zum Bestand dieser öffentlichen wissenschaftlichen Bibliothek. Sie waren seit Jahrzehnten jedem fachlich Interessierten zugänglich, sie sind seit jeher, schon lange, bevor die Amerika-Gedenkbibliothek sie erwarb, und bis in die unmittelbare Gegenwart von den an historisch-kritischer Philologie ernsthaft interessierten Wissenschaftlern auch genutzt worden, das waren stets wenige. Der letzte in der Reihe der Benutzer war 1992 Stefan Ormanns als der für den Text der Kleist-Briefe in der Edition des Deutschen Klassiker Verlages Verantwortliche, er hat Gewinn aus seinen Recherchen gezogen. Daß das KleistJahrbuch im gleichen Jahr an diese Archivalien erinnert hat, sei nur der Vollständigkeit halber erwähnt. Der erste der Benutzer jedoch war Helmut Sembdner. Seine Sammlungen 'Kleists Lebensspuren $\triangleleft$ und 'Kleists Nachruhm 1957 (!) erschienen sind, wären ohne Nutzung dieser Materialien gar nicht möglich 
gewesen. Sembdners Bände sind jedem an deutscher Literatur Interessierten ein Begriff, sie sind auch heute noch unentbehrlich. Sie sind freilich nicht vollständig, sie sind unzweckmäßig angeordnet, nicht fehlerfrei, vor allem aber sind sie nicht kommentiert. Diese Lage ist zweifellos unbefriedigend, sie bedarf eines ganz neuen Einsatzes, zu ihrer Verbesserung vermöchte die Sammlung Minde-Pouet jedoch nur wenig beizutragen. Kurz gesagt: Wer auf diesem Feld etwas ausrichten will, der muß einen grundsätzlichen Schritt noch vor die Sammeltätigkeit Minde-Pouets zurückgehen, indem er die Bücher und Handschriften in die Hand nimmt, die Minde-Pouet seinerseits benutzt hat. Aber das muß er selbst tun, denn fündig würde nur derjenige, der von sich aus bereits eigene Fragen mitbringt, der jedenfalls weiß, was er sucht. Es kann sein, daß er daraufhin etwas ganz anderes entdeckt, aber ein konkretes Ziel muß man bei archivalischer Suche nun einmal haben.

Der vorliegende Band des Jahrbuchs enthält zum Bedauern nicht nur seines Herausgebers keine Erörterungen zu Problemen der Kleist-Philologie im engeren Sinne. Nach dem Erscheinen der Abhandlung von Hans Zeller im Kleist-Jahrbuch 1992 besteht für den Augenblick keine Veranlassung, eine weitere Besprechung über die Kleist-Ausgabe von Reuß und Staengle, die im Verlag Stroemfeld / Roter Stern erscheint, zu vergeben. Bis jetzt hat man noch keines der Autographe Kleists in Angriff genommen, vor allem aber sind die Editionsprinzipien bisher nicht veröffentlicht worden. Jeder Rezensent bleibt somit auf Mutmaßungen angewiesen, was die Absichten der Herausgeber zu den spezifischen Problemen der Kleist-Überlieferung angeht. Die Herausgeber Reuß und Staengle haben sich über den Aufsatz Hans Zellers irritiert gezeigt. Dazu bestand kein spezieller Grund. Hans Zellers Ausführungen unterscheiden sich zwar in ihrer gründlichen Abwägung editorischer Prinzipienfragen, nicht aber in der Beurteilung der Verfahrensweise von den Rezensionen, es sind bisher neun, die Gerhard Schulz, Christian Wagenknecht, Hans-Gert Roloff, Klaus Kanzog, Wolfgang Wittkowski und ich in wissenschaftlichen Organen wie in Tageszeitungen veröffentlicht haben. Die Herausgeber verlangten Gelegenheit zu einer ausführlichen Entgegnung auf Hans Zeller im Kleist-Jahrbuch. Auf dieser Ebene hätte der Herausgeber des Jahrbuchs die Diskussion gerne fortgeführt. Auch der Vorstand der Heinrich-von-Kleist-Gesellschaft hätte es nachdrücklich begrüßt, wenn sich eine öffentliche wissenschaftliche Diskussion über diese Problematik im Kleist-Jahrbuch hätte ermöglichen lassen. Einer solchen Diskussion haben sich Reuß und Staengle dann entzogen, indem sie den Präsidenten der Heinrich-von-KleistGesellschaft auf den Artikel eines »befreundeten Journalisten « in einer Tageszeitung verwiesen. Auf diese Weise ist der Sache nicht gedient. In einer fachlichen, einer wissenschaftlichen Diskussion zählt nur, was der einzelne vorbringt, dafür hat er mit seinem Namen und seiner Person einzustehen. Publikationsorgane, Ämter und Institutionen führen dabei keine Stimme, auch die Heinrich-von-Kleist-Gesellschaft nicht. 